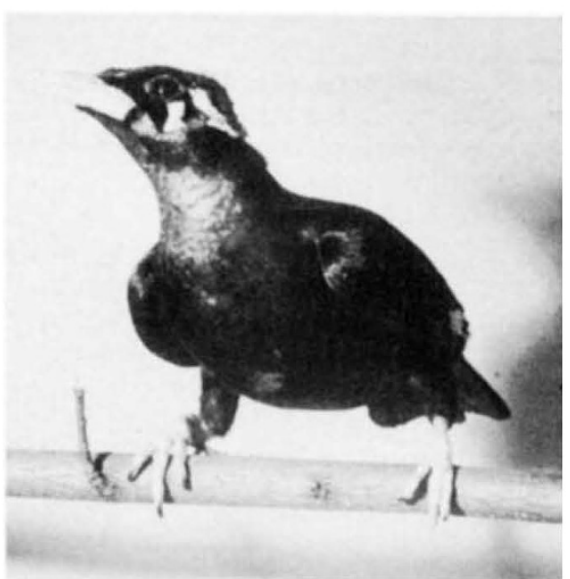

Indian hill mynah. (Photo: Zoological Society of London.)

with the capacity to imitate neighbours, indicate that these birds could potentially recognize a large number of different individuals and even recognize the locality of a bird which is not known individually. But why this should have evolved in mynahs so much further than in most other species of birds is still not known.

\section{ENZYMES}

\section{And Now Hysteresis}

from our Molecular Biology Correspondent

THE dwindling band of biochemists who have kept pace over the years with the ramifications of cooperativity, allosterism and induced-fit in complex enzymes, will not, one imagines, be best pleased by the suggestion that these all represent a gross and obvious oversimplification of the real situation. Such, however, is the message of an important new paper by Frieden (J. Biol. Chem., 245, 5788; 1970), which ends with the hope that the new concepts that it introduces will in the end be seen to simplify, rather than complicate, the interpretation of metabolic regulationa desire which will surely be weighed in its author's favour on the day of judgment.

Frieden makes the point that essentially all theories that treat of control of enzymic activity by non-competitive ligands, and the anomalous dependence of initial velocity on the concentration of such ligands or of substrate, make an unstated assumption. If the enzyme exists in conformational equilibrium between states of different enzymic characteristics, the possibility exists, and has so far been disregarded, that the rate of interconversion between the states may be slow relative to the formation of products. It is in fact already established that structural adjustments are indeed slow in many systems, most obviously perhaps in cases where they take the form of association or dissociation of the protein subunits. Frieden views such instances of slow equilibration as hysteresis effects, in which the measured catalytic rate after introduction of a modifying ligand, for example, is compounded of contributions from the state which is stable only in the absence of that ligand, and the state to which the ligand causes a slow transition. He formulates in these terms a number of models of varying complexity, in particular for systems involving the synthesis of a feedback inhibitor to which the enzyme responds in hysteretic fashion. Instead then of abruptly switching off, the enzyme buffers the effect of the change in inhibitor concentration. If the response were fast, the supply of metabolites produced by such an enzyme in a complex pathway would be instantaneously cut off, with perhaps dire results. There is no shortage of likely examples of hysteresis in action. One on which Frieden expatiates is the aspartate utilization scheme, worked out by Stadtman for $E$. coli, in which the feedback inhibition of homoserine dehydrogenase activity by threonine blocks also the production of methionione, and therefore normal growth. If the response to threonine were slow, on the other hand, the methionine concentration would remain sufficient for long enough to prevent the machinery from stalling while the threonine level once more regulates itself. Similarly the concentration of isoleucine is controlled at a remove by that of threonine, and the former is a feedback inhibitor of another activity, that of threonine deaminase. If the latter process is also slow, a rapid accretion of isoleucine will not bring about a catastrophic drop in the concentration of the other products dependent on the pathway, threonine, methionine and lysine.

Frieden finally makes the point that a change which is fast in relation to one pathway may be small on the time scale of another. Thus a slow change in the concentration of a metabolite may still be rapid compared with the hysteretic adjustment of the activity of an enzyme that it controls. One is persuaded by the numerous available examples of slow events in enzymatic systems that the hysteresis concept is likely to be of general importance, at any rate in complex, interlocking metabolic pathways, and it will no doubt be widely used to explain the inexplicable.

A good example in the current literature of conformational steps that are slow in relation to the rest of the catalytic scheme is to be found in the work of Shore and Gutfreund on liver alcohol dehydrogenase (Biochemistry, 9, 4655; 1970). Here the dissociation of NADH may be followed under appropriate conditions by the stopped-flow method,

\title{
Repair and DNA Polymerase
}

THE idea that the DNA polymerase of Escherichia coli, discovered by Kornberg and until recently thought to replicate DNA, is instead an enzyme concerned with the repair of damage in DNA, is lent further support by two articles in next Wednesday's Nature New Biology. It has previously been shown that the Cairns mutant of $E$. coli, which is defective in DNA polymerase activity when assayed in vitro, is unusually sensitive to the damage in DNA caused by irradiation with ultraviolet light, and Witkin and Klein and Niebch have used this mutant to try to pin down exactly which step in repair is affected by the lack of DNA polymerase.

When cells are irradiated with ultraviolet light, adjacent thymine bases in DNA are covalently linked together to form "thymine dimers". These dimers distort the structure of the DNA double helix, and many types of cell have now been shown to possess "excision-repair" systems which remove the dimers from DNA and replace them. But although DNA polymerase can excise the dimers from ultraviolet irradiated DNA in vitro, the excision of dimers has previously been shown to be normal in the mutant.

This suggests, of course, that DNA polymerase may be part of some other repair system. Witkin has studied the induction of mutations by ultraviolet light in the Cairns mutant; these muta- tions are caused by the attempts of another repair system, which is very errorprone, to fill the gaps which are left when DNA containing unexcised thymine dimers is replicated. But the number of mutations is the same in the mutant and in normal $E$. coli, so DNA polymerase cannot be part of the repair system which is error prone.

Bacteriophages which have been damaged by ultraviolet irradiation can be repaired by the host excision-repair system during infection of bacterial cells. Klein and Niebch show that phages $\varphi \times 174$ and $\lambda$ are repaired less efficiently by the Cairns mutant than by normal bacteria. This means that DNA polymerase must be part of the excisionrepair mechanism which acts on bacteriophages. When the host cells have been mutated in one of the genes coding for the enzymes of this system, addition of a mutation in the DNA polymerase gene does not make repair any less efficient. This confirms that DNA polymerase belongs to the same system as the enzyme coded by the other mutated gene.

The only firm conclusion to emerge from these data is that DNA polymerase is concerned with excision-repair, but acts at some stage after the thymine dimers have been excised. Just what it does, and why this is important for proper repair of damaged bacterial or bacteriophage DNA, remains to be established. 